\title{
A PERCENTAGE BRIDGE.
}

BY HERSCHEL C. PARKER.

There are many cases in the measurement of resistance that require the exact determination of comparatively small differences. This is true when standard ohms are compared, when a standard rheostat is calibrated by comparing directly with a standard ohm, in the measurement of temperature coefficients, etc.

For the comparison of standard resistances of the same value, the Carey-Foster method, using the "commutator bridge," furnishes a very precise and satisfactory means of measurement.

If, however, a rheostat in which the coils are arranged in sets of $1,2,3,4$ or $1,2,2,5$ is to be calibrated against a standard ohm, the problem becomes an exceedingly difficult one. In fact two rheostats have to be calibrated at the same time and the corresponding calculations are very involved.

The method known as "substitution in the bridge" (described in "A Systematic Treatise on Electrical Measurements") is perfectly adapted to the adjustment of the coils of a standard rheostat of any pattern whatever by comparison with a standard ohm, but requires the use of interpolation resistances and the observation of galvanometer deflections when a table of corrections is to be made out for a rheostat already adjusted.

The method devised by the writer, which may be called the "percentage method" and the apparatus employed the "percentage bridge," while based on this method, differs from it radically. It is a zero method, makes use of readings on the bridge wire in place of galvanometer deflections, and does away entirely with the use of interpolation resistances.

It seems to possess all the advantages of the Carey-Foster method, while the apparatus required is much simpler using only 
4 mercury cups in place of the 20 mercury cups of the complicated commutating device usually made use of. At the same time it gives the great range of measurement of the method of percentage differences.

The arrangement of the apparatus is shown in Fig. 1.

The "percentage coils" $C O$ are joined to the ends of the bridge wire $A B$ and these coils are so adjusted that when resistance in one arm of the bridge is changed by an amount $\theta$ the displacement on the bridge wire $=\lambda$.

Let $L=1 \mathrm{ohm}$ and $R=1 \mathrm{ohm}$. Then $(C+A)-\lambda:(C+B)+\lambda:: L: R+\theta$.

Now when $\theta=.01 \mathrm{ohm}$, adjust the coils $C O$ so that $\lambda=100$ divisions of the bridge wire, then 1 division $=.01 \%$ (approximately).

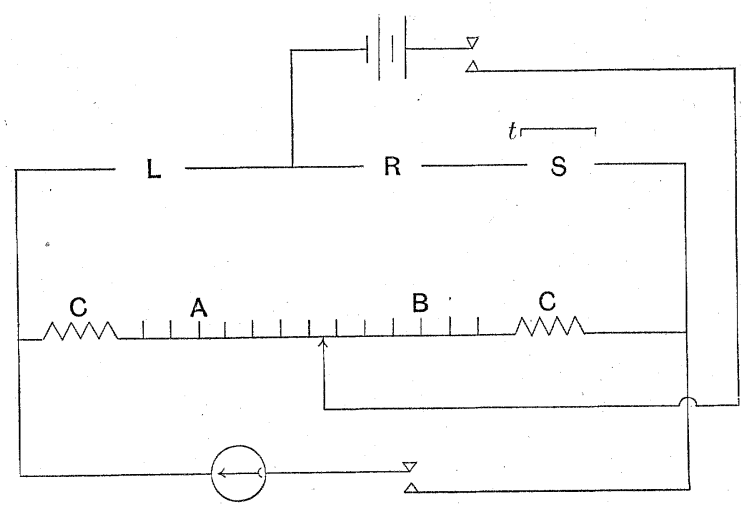

FIG. 1.

If $L=10 \mathrm{ohms}$ and $R=10 \mathrm{ohms}$, when $\lambda=100$ divisions $\theta=0.1 \mathrm{ohm}(1 \%$ of $10 . \mathrm{ohms})$ and 1 division is again $.01 \%$.

This being true for any values of $L$ and $R$, the bridge when once adjusted will always read in percentage.

The value of the "percentage coils" for any given value of the wire $A B$ can be calculated as follows:

Let $A+B=1 \mathrm{ohm}=1000$ scale divisions, and the ratio of the change of resistance $=100 / K$.

Suppose the position of balance to be at the center of the bridge wire, then $A=B$ and

$$
\begin{array}{lr}
A+C=B+C=X . & \text { Whence } \frac{X-\lambda}{X+\lambda}=\frac{100}{K}, \\
K X-100 X=K \lambda+100 \lambda, & X=\frac{\lambda(K+100)}{K-100} .
\end{array}
$$


If $K=101$ and $\lambda=0.1 \mathrm{ohm}(100$ divisions $)$, $X=\frac{0.1(101+10(1)}{101-100}=20.1$ and $C=X-A=20.1-.5=$ $19.6 \mathrm{ohms}$.

To find how nearly this value of $C$ will give the true difference in percentage for any other value of $\lambda$ we may substitute in the equation $\frac{X-\lambda}{X+\lambda}=\frac{100}{K}$, thus when $\lambda=.001$ ohm (1 division) and $K$ should equal 100.01, $\frac{20.1-.001}{20.1+.001}=\frac{100}{K}, K=$ 100.00995 , which gives an error of only $.00005 \%$, and when $\lambda=.01 \mathrm{ohm}$ (10 divisions), $K=100.09855$, an error of only $.00045 \%$. These errors are, of course, quite beyond the errors of observation:

Thus we may have a bridge that will read in differences of percentage with the greatest accuracy from considerably above $1 \%$ to differences in resistance as small as .001\%.

By taking different values for $\lambda$ and $K$ in the equation $X=\frac{\lambda(K+100)}{K-100}$, and then calculating from the values obtained for $X$ the corresponding values for $K$ from the equation. $\frac{X-\lambda}{X+\lambda}=\frac{100}{K}$, it is found that the best ratio of the bridge wire to the "percentage" coils for all usual cases of measurement is $1: 19.6$.

If it is desired to have 1 division of the bridge wire $=.001 \%$, then the ratio should be, $1: 200.5$.

If the point of balance is not exactly at the center of the bridge wire the error introduced is very small. Thus, suppose in the case where 1 division $=.01 \%$ the position of balance was 100 divisions $(0.1 \mathrm{ohm})$ from the center, then when balanced the ratio is, 20.2: $20.0:: 1.01: 1$, and when $\lambda=100$ divisions $(0.1$ $\mathrm{ohm}$ ) the ratio is $20.3: 19.9:: 1.0201: 1$. Now $1.0201-1.0100=$ .0101 , an error of only $.01 \%$.

This error may, of course, be eliminated if necessary by adding compensating wires to either arm of the bridge when the point of balance is a considerable distance from the center.

This form of bridge is of great service in the laboratory when exact and rapid comparisons are desired. An example will best show the method of employment. 
Queen Rheostat No. 1 compared with Standard $0 h m=$ 1.0005 at $19^{\circ} \mathrm{C}$.

$\begin{array}{ccccc}\begin{array}{c}\text { Pusitions on } \\ \text { Balance. }\end{array} & \begin{array}{c}\text { Bridge Wire } \\ \text { Substitution. }\end{array} & \begin{array}{c}\text { Difference } \\ \text { in } \%\end{array} & \begin{array}{l}\text { Correct } \\ \text { Value. }\end{array} & \begin{array}{c}\text { No. of } \\ \text { Coil. }\end{array} \\ 500 & 495 & -.05 & 1.0000 & 1 \\ 426 & 435 & +.09 & 2.0023 & 2 \\ 409 & 395 & -.14 & 2.9986 & 3\end{array}$

This method is especially adapted to the determination of temperature coefficients, for the change from the position of balance divided by the difference in temperature gives at once the coefficient. Since a change in resistance of .001\% can easily be detected, the temperature coefficient when a difference of temperature of only 1 degree is employed can be accurately measured. This bridge also gives a convenient means for determining small changes in temperature. If a wire whose temperature coefficient $=0.2 \%$ is balanced in one arm of the bridge, then it is possible to measure a change of temperature $=.005$ degree.

The "percentage bridge" is so easy to construct and adjust, being, in fact, only an ordinary bridge wire with the addition of the "percentage" coils, that it seems possible it may be found ueeful in many electrical laboratories when the precise comparison of standard obms or the calibration of standard rheostats is required. 


\section{Discussion.}

The President:- There already exist many methods of measuring resistances, but there are none too many, as most of us have found frequently, to our sorrow, that often the very resistance we want to measure cannot be measured as well as we would like, by the methods that are known to us. The present method points out the importance of measuring by percentages, and I think that principal ought to be recognized more than it is, because in making such calibrations it is not the absolute values of the errors that we are after, but the percentage value, and a method of finding this directly cannot fail to be of interest. The paper is open for discussion.

PRof. Goldsborodgh :-I have been very much interested indeed in reading this paper, and in listening to its presentation to-day. I very gladly welcome at all times anything that tends to reduce labor in calibrating instruments of any kind-anything that tends to greater simplicity. The greater the simplicity of the method the greater are the chances of ultimate accuracy in our results. This method commends itself, especially in view of the fact that it is a zero method, and we all know the value of zero methods. A zero method, in which a galvanometer is used may be said to be analogous to a contact method in which we adjust for maximum noise instead of trying to observe a point of silence. It gives us a direct indication that we have reached the desired result. Very frequently it is a very difficult matter to make a galvanometer which is sensitively adjusted, operate satisfactorily, unless we go to a great deal of trouble. At our own University we find that often the street railway currents affect our apparatus, when the street car line is not closer at any point than a quarter of a mile; and I have no doubt that in laboratories in cities which are less favorably located, experimenters experience much difficulty in getting their galvanometers to behave properly. Under these conditions a zero method is all the more valuable, as it eliminates the necessity of taking a reading of a given deflection. We have found instances in the case of very delicate readings that we have been making recently at the Purdue University, that it has been necessary for the experimenters to do all their work between 1 o'clock at night and early morning before the street car lines start up. We reach a time only then when our instruments can be very delicately adjusted.

I very gladly welcome any method which tends to simplicity and greater accuracy with less wear and tear on the human frame; so I think those of us who are in charge of laboratories will welcome this particular device for standardizing our resistance coils as something to be highly commended.

The President :-Is there any further discussion. If not, the Secretary has some announcements to make.

The Sedretary :--To-day's session ends with the paper just read. I take pleasure in announcing that the Local Committee 
has arranged for a "Smoker," which by the courtesy of the Manufacturers' Club will be held at its rooms, No. 1405 Walnut Street.

Mr. Charles A. Bragg, Chairman of the Local Committee then read letters of invitation to the Institute to visit Cramp's Shipyard, the Baldwin Locomotive Works, and the Franklin Institute.

The meeting then adjourned to Thursday, May 17 th. 